\title{
Pathological Evidence of Cardiac Papillary Fibroelastoma in a Retrieved Intracranial Embolus
}

\author{
Ahmed Itrat, Pravin George, Zeshaun Khawaja, David Min, Megan Donohue, Dolora Wisco, \\ E. Rene Rodriguez, Carmela D. Tan, Muhammad Shazam Hussain
}

Keywords: Embolism, fibroelastoma ischemic stroke, papillary

doi:10.1017/cjn.2014.114

Can J Neurol Sci. 2015; 42: 66-68

Approximately one-quarter of all cardiac tumors present with systemic embolization. ${ }^{1}$ Cardiac papillary fibroelastomas (CPF) represent a potential embolic source, accounting for $8 \%$ of all primary cardiac tumors, second in frequency after cardiac myxomas. ${ }^{2}$ Most case reports describe papillary fibroelastoma as a potential source for stroke, ${ }^{3}$ but histopathological evidence of actual tumor fragment causing a stroke is lacking. We describe a case of histologically confirmed CPF mechanically retrieved from a cerebral artery during an emergent cerebral endovascular procedure with subsequent discovery of the $\mathrm{CPF}$ in the heart, providing in vivo demonstration of intracranial embolization of this cardiac tumor.

\section{CASe Report}

A 62-year-old Caucasian man with history of hypertension, hyperlipidemia, coronary artery disease, and tobacco use presented to an outside hospital with acute onset of right-sided weakness and global aphasia. His National Institutes of Health Stroke Scale score was 24. A CT scan of the brain showed no hemorrhage, and intravenous tissue plasminogen activator was started 3.5 hours after symptom onset without improvement. An MRI and a magnetic resonance angiogram of the brain showed an M1 middle cerebral artery occlusion with diffusion restriction limited to the deep structures and significant mismatch between diffusion and perfusion imaging.

The patient was transferred to our institution three hours later with no clinical change. Repeat MRI and magnetic resonance angiogram were stable. Because of the clinical diffusion and diffusion perfusion mismatch, he underwent emergent intra-arterial stroke therapy. Mechanical thrombectomy was performed using a Solitaire stent retriever (Covidien Inc, Irvine, CA). Thrombolysis in cerebral infarction grade $2 \mathrm{~b}$ was achieved after a single attempt. The stent retriever was removed with embolus, which was noted to have a "jellylike" texture (Figure 1). Pathology review of the embolus revealed a papillary mass consistent with a papillary fibroelastoma with no associated thrombus (Figure 2A, B).

Postprocedural brain MRI showed a stable infarct size. Stroke workup including a transthoracic echocardiogram was normal. A transesophageal echocardiogram was attempted, but was not tolerated by the patient. He was discharged to a rehabilitation center. A few weeks later, the transesophageal echocardiogram was reattempted, which revealed a mobile left atrial mass measuring $23 \times 16 \times 14 \mathrm{~mm}$. The patient was admitted for open heart surgery and the mass was excised from the left atrium and confirmed to have matching microscopic characteristics of the inciting embolism (Figure 2C). The patient continued to improve, and at his three-month follow-up was ambulating (National Institute of Health Stroke Scale =10; modified Rankin Score =3).

\section{Discussion}

Our case represents a rare demonstration of in vivo retrieval of histologically confirmed CPF, which was mechanically

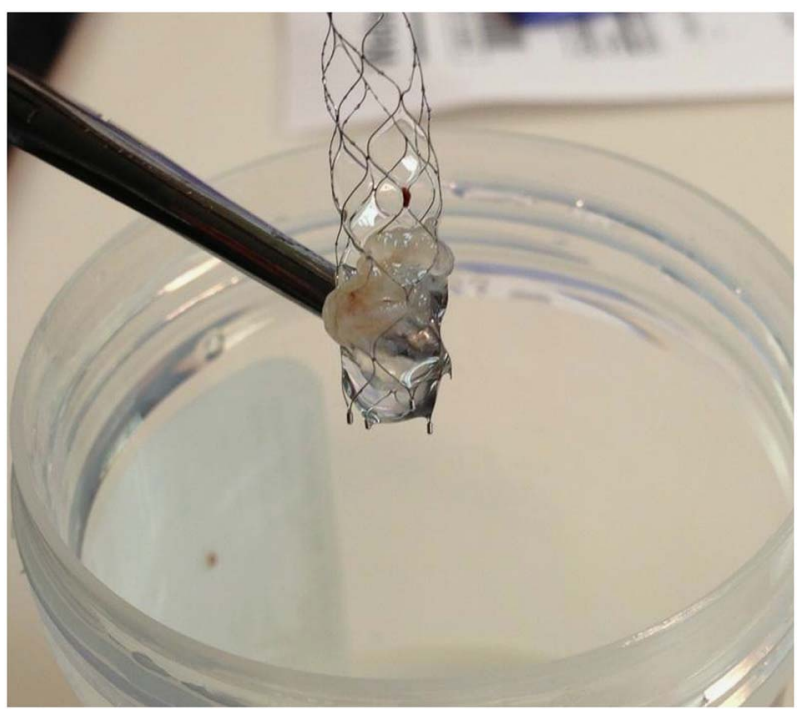

Figure 1: Gross picture of an embolus extracted using a Solitaire stent retriever showing a tan white tissue with gelatinous consistency.

From the Cerebrovascular Center, Neurological Institute (AI, PG, ZK, MD, DW, MSH), Heart and Vascular Institute (DM), and Department of Anatomic Pathology (ERR, CDT), Cleveland Clinic, Cleveland, Ohio.

Received July 24, 2014. Final Revisions Submitted SePtember 29, 2014. Correspondence to: Muhammad Shazam Hussain, 9500 Euclid Ave, S80, Cleveland Clinic, Cleveland, $\mathrm{OH}$ 44106. Email: hussais4@ccf.org 

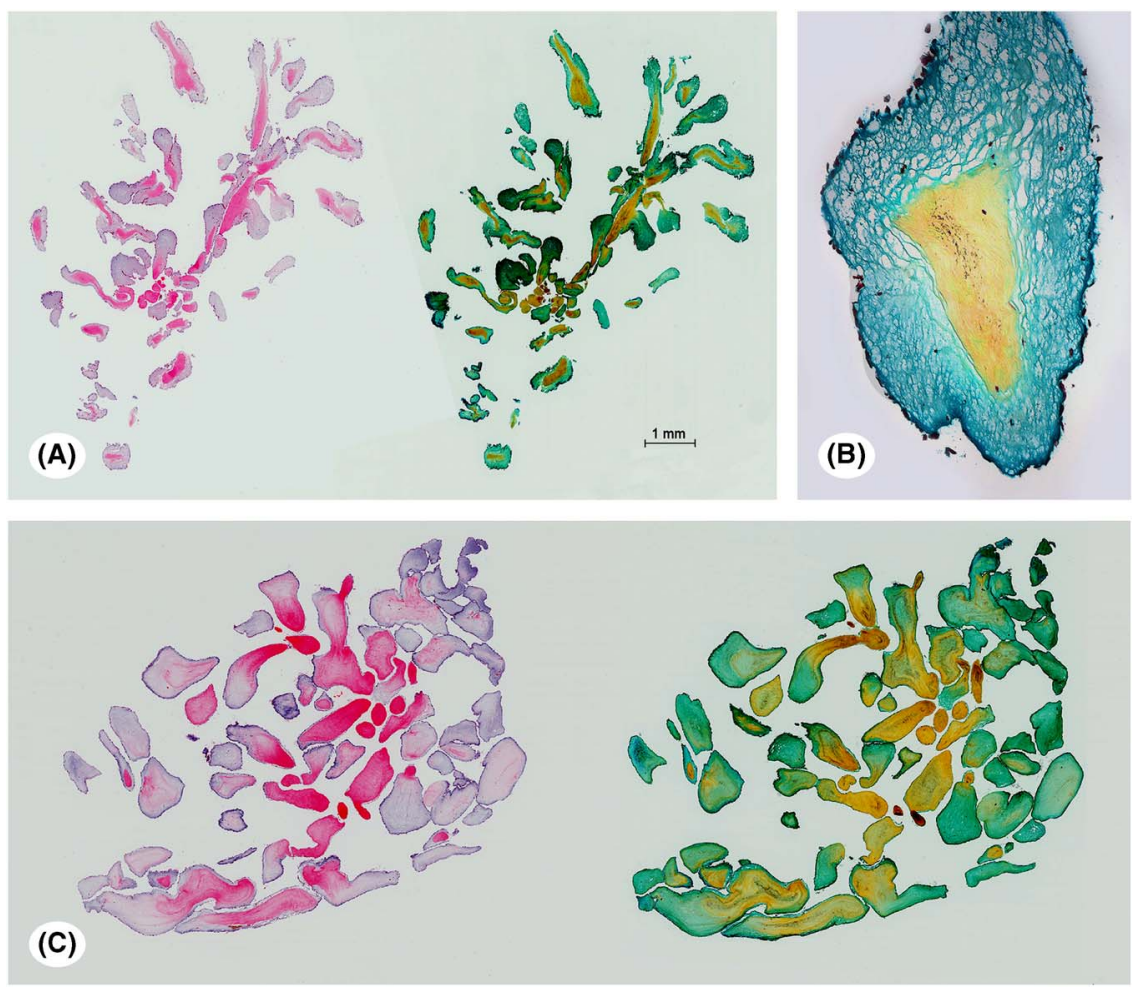

Figure 2: (A) Scanning magnification microphotograph of the extracted embolus shows a benign tumor with papillary fronds (left, hematoxylin-eosin; right, Movat pentachrome stain; original magnification, $40 \times$ ). (B) The embolic papillae have a collagenous core (yellow) containing elastin (black speckles) and lined by endothelial cells. The tumor is rich in mucopolysaccharide (blue green), giving it a gelatinous consistency (Movat pentachrome stain; original magnification, $400 \times)$. (C) The excised cardiac mass is structurally and histologically similar to the embolus (left, hematoxylin-eosin; right, Movat pentachrome stain; original magnification, $40 \times$ ).

retrieved from the middle cerebral artery during an emergent cerebral endovascular procedure, providing direct evidence of the embolization potential of this tumor. This embolic risk has been recently described in several case reports. ${ }^{4-6}$ Primary cardiac tumors are rare, accounting for $<1 \%$ of ischemic strokes, ${ }^{7}$ are often discovered on follow-up echocardiogram after a stroke. CPF mostly originate from the valvular endocardium $(85 \%)$ and involve the aortic (29\%), mitral (25\%), tricuspid (17\%), and pulmonary valves $(13 \%)$ in decreasing order of frequency. ${ }^{8}$ They are noted to have multiple frond-like projections on an underlying pedicle, giving them the appearance of a sea anemone. They are usually solitary, but some patients have multiple tumors throughout the left-sided chambers. The symptomatic presentation of CPF includes ischemic stroke, syncope, congestive heart failure, and ventricular arrhythmias. ${ }^{5}$ The origin of these tumors is unclear. Thoracic radiation or cardiac surgeries may be potential causes, and the tumors may also be related to organizing thrombi resulting from the presence of fibrin, hyaluronic acid, and laminated elastic fibers. ${ }^{9} \mathrm{CPF}$ have no known malignant potential but are considered dangerous because of their propensity to embolize. Left-sided tumors present with systemic embolization, whereas right-sided tumors remain predominantly asymptomatic until large enough to interfere with intracardiac blood flow, alter hemodynamic function, or induce arrhythmias.
Management of CPF has remained controversial. Although early tumor removal has been proposed in some instances, an incidental finding of $\mathrm{CPF}$ on echocardiogram does not necessarily lead to a surgical referral. One of the reasons is the uncertainty of risk of embolization, along with the fear of surgical complications. Elbardissi et al demonstrated a $25 \%$ rate of embolization of all cardiac tumors, but did not stratify this risk according to tumor type. ${ }^{1}$ Once diagnosed following a stroke, symptomatic CPF management is removal of tumor to prevent further embolic events. The outcome of such strokes is dependent on the extent and size of the infarct, and there are not enough data to predict if clot composition itself predicts the outcome of stroke. Timing of cardiac surgery is of critical importance because a large-territory infarct is at risk of bleeding during cardiopulmonary bypass in the acute setting, whereas waiting too long may lead to further embolic events. Surgical management follows a period of watchful waiting, and the patient's modifiable stroke risk factors are addressed in the interim to prevent any further stroke.

Advancement in endovascular management of stroke has allowed us to identify potential sources of emboli by routine histopathological analysis of extracted clot products. Pathologic examination may allow the identification of primary cardiac tumors, as evidenced in this case. Existing studies suggest such tumors should be surgically removed to prevent future embolic risks. 


\section{REFERENCES}

1. Elbardissi AW, Dearani JA, Daly RC, et al. Embolic potential of cardiac tumors and outcome after resection: a case-control study. Stroke. 2009;40(1):156-62.

2. Burke A, Virmani R. Classification and incidence of cardiac tumors. In: Tumors of the heart and great vessels. Atlas of tumor pathology. Third series. Washington, DC: Armed Forces Institute of Pathology; 1996. p. 1-12.

3. Gallanagh S, Walters M, Mallon E, et al. Fibroelastoma of the mitral valve as a cause of embolic cerebral infarction. BMJ Case Rep. $2011 \mathrm{Jul} ; 15: 2011$.

4. Vaskelyte L, Risteski P, Bertog S, et al. A case of a fibroelastoma and patent foramen ovale in a patient with prior stroke. Cardiovasc Diagn Ther. 2014 Feb;4(1):47-9.

5. Santos AF, Pinho J, Ramos V, Pardal J, et al. Stroke and cardiac papillary fibroelastoma: mechanical thrombectomy after thrombolytic therapy. J Stroke Cerebrovasc Dis. 2014 May-Jun; 23(5):1262-4.

6. Tejada J, Galiana A, Balboa O, Clavera B, et al. Mechanical endovascular procedure for the treatment of acute ischemic stroke caused by total detachment of a papillary fibroelastoma. J Neurointerv Surg. 2014 Jul;6(6):e37.

7. Hart RG, Albers GW, Koudstaal PJ. Cardioembolic stroke. In: Ginsberg MD, Bogousslavsky J, editors. Cerebrovascular disease: pathophysiology, diagnosis and management. London: Blackwell Science; 1998. p. 1392-429.

8. Grinda JM, Couetil JP, Chauvaud S, D'Attellis N, et al. Cardiac valve papillary fibroelastoma: surgical excision for revealed or potential embolization. J Thorac Cardiovasc Surg. 1999;117:106-10.

9. Howard RA, Aldea GS, Shapira OM, et al. Papillary fibroelastoma: Increasing recognition of a surgical disease. Ann Thorac Surg. 1999;68:1881-5. 